\title{
KARP HARDNESS OF DIGRAPH COLORING WITHOUT MONOCHROMATIC DICYCLE
}

\author{
THINH D. NGUYEN
}

\begin{abstract}
We show that it is hard to color a digraph with few colors such that there is no monochromatic dicycle.
\end{abstract}

\section{Definition And Claim}

Definition 1. Digraph Coloring Problem:

Input: An oriented graph $G=(V, A)$ and a natural number $k$

Output: YES if it is possible to color all vertices of $V(G)$ by $k$ colors such that no dicycle is monochromatic, otherwise No

Our problem is NP-complete by a reduction from NAE-3SAT. In fact, both the undirected and directed (oriented) versions are NP-complete. First, we will describe the reduction for the undirected version. Then, we show how to orient all the edges of the undirected graph to obtain a digraph to finish the reduction for the directed version.

Claim 2. We have that Nae-3Sat $\leq{ }_{p}$ Digraph Coloring Problem

\section{REDUCTION FOR UNDIRECTED VERSION}

Given an instance of NAE-3SAT, we will construct an undirected graph such that there exists a solution for the NAE-3SAT instance if and only if it is possible to color the vertices of this graph by 2 colors such that no cycle is monochromatic.

For each clause $j$, create a $K_{3}$ cycle each vertex of which corresponds to one literal of clause $j$.

Now, for each pair of opposite literals (one positive and one negative literal of the same variable) $l_{j m}=x_{i}$ and $l_{k n}=\neg x_{i}$, we connect them by a gadget as follows. (Here, $l_{a b}$ is literal $b$ th of clause $a$, where $1 \leq b \leq 3$ )

The gadget to connect $l_{j m}$ and $l_{k n}$ is described now:

- Connect $l_{j m}$ and $l_{k n}$ by an edge

- Create 3 new vertices $a, b, c$ (each gadget has its own three vertices like these)

- Connect these 3 vertices $a, b, c$ to form a $K_{3}$ cycle

- Connect each of $a, b, c$ with both $l_{j m}$ and $l_{k n}$

Call the obtained undirected graph $G$. Set $k=2$. The reduction returns the instance $(G, k)$ of our problem. Now, we prove the correctness of our reduction (for the undirected version).

Key words and phrases. graph, coloring, digraph, chromatic, dicycle.

Perebor. 
If the given NAE-3SAT is satiafiable, then given a solution, one can color all the TRUE literal-vertices RED and all the FALSE literal-vertices GREEN. For each gadget described above, we color $a$ and $b$ by RED and $c$ by GREEN. Next, we show that this is a solution to the produced instance of our problem.

Clearly, we use only $k=2$ colors as required. For each $K_{3}$ corresponding to one of the clauses, its 3 vertices cannot be colored by the same color, because a solution to an NAE-3SAT instance would make every clause containing both TRUE literal (colored RED) and FALSE literal (colored GREEN).

For each gadget of 2 opposite literals $l_{j m}$ and $l_{k n}$, we have that these 2 are colored by different colors. So, every cycle (contained in this gadget) that contains both these two vertices is already non-monochromatic. Only one other cycle (contained in this gadget) is the $K_{3}$ of $a, b$ and $c$. But as mentioned above, $a$ is colored RED and $c$ is colored GREEN. All other possible cycles in $G$ need to cross from a $K_{3}$ clause to other $K_{3}$ clause by passing through a gadget. But, to pass through a gadget of, say $l_{j m}$ and $l_{k n}$ (colored by different colors), such a cycle has to be non-monochromatic.

Conversely, if we can color $G$ 's vertices by 2 colors without making any cycle monochromatic, then we will now show that the given NAE-3SAT instance is satiafiable.

For each literal-vertex $l_{j m}=x_{i}$, if $l_{j m}$ is colored RED, we assign $x_{i}$ to TRUE, otherwise assign it to FALSE.

For each literal-vertex $l_{j m}=\neg x_{i}$, if $l_{j m}$ is colored RED, we assign $x_{i}$ to FALSE, otherwise assign it to TRUE.

Since each clause is a $K_{3}$ cycle, it has to contain both RED literal-vertex (assigned to TRUE) and GREEN literal-vertex (assigned to FALSE).

It is left to prove the consistency of the above mentioned assignment. This is guaranteed by the gadgets. Suppose to the contrary that there exist two oppisite literals $l_{j m}$ and $l_{k n}$ colored by the same color, that w.l.o.g. we can assume to be RED. Then it is impossible to properly color $a, b$ and $c$. Indeed, since $a, l_{j m}$ and $l_{k n}$ form a cycle, we deduce that $a$ must be colored GREEN. Similarly, $b$ and $c$ are also colored GREEN. But, now the cycle $(a, b, c)$ is monochromatic. Thus, the above assignment is consistent. And we obtain a solution to the given NAE-3SAT.

\section{REDUCTION FOR DIRECTED VERSION}

We use exactly the same above reduction. Now, we have to orient all the edges of $G$ to obtain a digraph. For each, $K_{3}$ clause cycle, we are free to choose one of two ways to turn it into a directed cycle (dicycle). For each gadget of two opposite literals $l_{j m}$ and $l_{k n}$, we can orient to have an $\operatorname{arc}\left(l_{j m}, l_{k n}\right)$ (the other way can be finished similarly). Now, we orient to make 3 cycles: $\left(l_{j m}, l_{k n}, a\right),\left(l_{j m}, l_{k n}, b\right),\left(l_{j m}, l_{k n}, c\right)$. Then, we make one more cycle, namely $(a, b, c)$. By orienting like this, we can use all the above arguments for the undirected version. Note that the possible dicycles that cross from clause to clause still have to be non-monochromatic.

\section{Conclusion}

Garey and Johnson [1] shape their theory based on previous primal works of Cook, Levin and Karp. Johnson [2] moves on with the guide to this theory. As long as we study a mathematical conjecture, we should encourage ourselves to have enough labour hours on popular math books like these. Then, reading some articles 
KARP HARDNESS OF DIGRAPH COLORING WITHOUT MONOCHROMATIC DICYCLE 3

on theory of computing like [3] is a good practice. Only after that, could we think of the ultimate final for all mathematical sciences.

\section{REFERENCES}

1. Michael R. Garey, David S. Johnson, Computers and Intractability: A Guide to the Theory of NP-Completeness

2. David S. Johnson, The NP-Completeness Column: An Ongoing Guide

3. Phan Dinh Dieu, Le Cong Thanh, Le Tuan Hoa, Average Polynomial Time Complexity of Some NP-Complete Problems, Theor. Comput. Sci. 46(3): pp.219-237 (1986)

Email address: kosmofarmer@yandex.com 